\title{
Bootstrap prediction for returns and volatilities in GARCH models
}

\author{
Lorenzo Pascual $^{\mathrm{a}}$, Juan Romo ${ }^{\mathrm{b}}$, Esther Ruiz ${ }^{\mathrm{b}, *}$ \\ ${ }^{a}$ Hidro-Cantábrico, Serrano Galvache 56, Centro Empresarial Parque Nort, 28033 Madrid, Spain \\ ${ }^{\mathrm{b}}$ Departamento de Estadística, Universidad Carlos III de Madrid, \\ C/Madrid 126, 28903 Getafe, Spain
}

Available online 18 January 2005

\begin{abstract}
A new bootstrap procedure to obtain prediction densities of returns and volatilities of GARCH processes is proposed. Financial market participants have shown an increasing interest in prediction intervals as measures of uncertainty. Furthermore, accurate predictions of volatilities are critical for many financial models. The advantages of the proposed method are that it allows incorporation of parameter uncertainty and does not rely on distributional assumptions. The finite sample properties are analyzed by an extensive Monte Carlo simulation. Finally, the technique is applied to the Madrid Stock Market index, IBEX-35.
\end{abstract}

Keywords: Time series; Non-Gaussian distributions; Nonlinear models; Resampling methods

\section{Introduction}

Financial market participants have an increasing interest in prediction intervals for future returns as measures of uncertainty. For example, in the area of financial risk management, it is important to provide density forecasts of portfolio prices and to track certain aspects of these densities such as value at risk (VaR); see, for example, Bollerslev (2001) and Engle (2001). On the other hand, accurate predictions of future volatilities are critical for the implementation and evaluation of asset and derivative pricing theories as well as trading and

\footnotetext{
* Corresponding author. Tel.: +34 916249851; fax: +34916249849.

E-mail address: ortega@est-econ.uc3m.es (E. Ruiz).
} 
hedging strategies. It is by now well documented in the literature that the volatility of financial returns evolves over time. Generalized autoregressive conditionally heteroscedastic (GARCH) models, originally introduced by Bollerslev (1986), provide dynamic prediction intervals which are narrow in quiet times and wide in volatile periods. However, despite the extensive literature related with GARCH models, relatively little attention has been given to the construction of prediction intervals for GARCH models. Furthermore, the literature on volatility prediction has traditionally dealt with point forecasts without considering any measure of the uncertainty associated to forecasting future volatilities. The interest of having this measure has been put forward, among many others, by Baillie and Bollerslev (1992), Shephard (1996), Andersen and Bollerslev (1998), Andersen et al. (2001), Engle and Patton (2001) and Tsay (2002). The few procedures available in the literature to obtain prediction intervals for future volatilities are mainly Bayesian and need to assume a particular distribution for the errors; see, for example, Jacquier et al. (1994).

In this paper, we propose to use bootstrap methods to obtain prediction densities of future returns and volatilities generated by GARCH models. Our proposal is a generalization of the procedure proposed by Pascual et al. (2004) for linear autoregressive integrated moving average (ARIMA) models. The resulting prediction intervals for returns and volatilities incorporate the uncertainty due to parameter estimation without distributional assumptions on the sequence of innovations. Miguel and Olave (1999) have also proposed a bootstrap procedure to obtain prediction intervals for future observations generated by ARCH models but their intervals do not incorporate the parameter uncertainty. Consequently, their proposal does not allow to construct prediction intervals for one-step ahead future volatilities.

The paper is organized as follows. Section 2 describes the main properties of GARCH processes and predictions. In Section 3, we present the proposed resampling procedure to estimate prediction densities and intervals for returns and volatilities. Its finite sample behavior is analyzed in Section 4, which reports the results of an extensive Monte Carlo simulation study. Section 5 presents an application with real financial data. Finally, the conclusions appear in Section 6.

\section{The $\operatorname{GARCH}(1,1)$ model}

The GARCH $(1,1)$ model provides a simple representation of the main dynamic characteristics of returns of a wide range of assets and it is extensively used to model them. Hence, although it could not be the optimal model for volatility forecasting in any given series, it serves as a natural benchmark for the forecast performance of heteroscedastic models. For simplicity, we concentrate on it hereafter. However, the bootstrap procedure proposed in the following section can be directly generalized to general $\operatorname{GARCH}(p, q)$ processes. A $\operatorname{GARCH}(1,1)$ model is given by

$$
\begin{aligned}
& y_{t}=\sigma_{t} \varepsilon_{t}, \\
& \sigma_{t}^{2}=\omega+\alpha y_{t-1}^{2}+\beta \sigma_{t-1}^{2}, \quad t=1, \ldots, T,
\end{aligned}
$$

where $\varepsilon_{t}$ is a white noise process with unit variance, $\sigma_{t}$ is a stochastic process known as volatility and assumed to be independent of $\varepsilon_{t}$, and $\omega, \alpha$ and $\beta$ are unknown parameters that 
satisfy $\omega>0, \alpha \geqslant 0, \beta \geqslant 0$ to ensure the positivity of the conditional variance. The process $y_{t}$ is covariance stationary if $\alpha+\beta<1$. Nelson (1990) shows that $y_{t}$ is strictly stationary if $E\left[\log \left(\beta+\alpha \varepsilon_{t}^{2}\right)\right]<1$. Note that $\sigma_{t}^{2}$ is observable with information available at time $t-1$ and, consequently, given the assumptions on the distribution of $\varepsilon_{t}$, the conditional mean of $y_{t}$ is zero and $\sigma_{t}^{2}$ is the conditional variance. Moreover, the conditional distribution of $y_{t}$ coincides with the distribution of $\varepsilon_{t}$.

Alternatively, the conditional variance can also be expressed as a function of past observations as follows:

$$
\sigma_{t}^{2}=\frac{\omega}{1-\alpha-\beta}+\alpha \sum_{j=0}^{\infty} \beta^{j}\left(y_{t-j-1}^{2}-\frac{\omega}{1-\alpha-\beta}\right) .
$$

The predictor of $y_{T+k}$, given observations of the process up to time $T, Y_{T}=$ $\left\{y_{1}, y_{2}, \ldots, y_{T}\right\}$, is zero and its conditional mean square error (MSE) is given by

$$
E_{T}\left(\sigma_{T+k}^{2}\right)=\frac{\omega}{1-\alpha-\beta}+(\alpha+\beta)^{k-1}\left(\sigma_{T+1}^{2}-\frac{\omega}{1-\alpha-\beta}\right) .
$$

If $\varepsilon_{t}$ is further assumed to be a Gaussian process, then $y_{t}$ is conditionally Gaussian, and one-step ahead forecast errors are normally distributed. Therefore, $(1-\gamma) \%$ prediction intervals of $y_{T+1}$ are given by $\pm z_{\gamma / 2} \sigma_{T+1}$, where $z_{\gamma / 2}$ is the $\gamma / 2$-quantile of the standard normal density. On the contrary, the prediction error distribution when forecasting $k$-periods ahead for $k>1$ is not normal even if $\varepsilon_{t}$ is Gaussian. However, the usual approximation to the $(1-\gamma) \%$ prediction intervals of returns $y_{T+k}$ for $k>1$ is given by

$$
\pm z_{\gamma / 2} E_{T}\left(\sigma_{T+k}\right) \text {. }
$$

Alternatively, Baillie and Bollerslev (1992) propose an improvement of the intervals in (4) based on Cornish-Fisher expansions making parametric assumptions on the distribution of $\varepsilon_{t}$. However, the prediction error distribution is only known for the $\operatorname{GARCH}(1,1)$ model and it seems difficult to generalize it to general $\operatorname{GARCH}(\mathrm{p}, \mathrm{q})$ processes. Furthermore, Baillie and Bollerslev (1992) point out that the resulting intervals do not take into account the uncertainty due to parameter estimation and the fact that it could be of interest to know whether this uncertainty may have an effect on their properties.

With respect to the prediction of future values of volatilities, the point predictor of $\sigma_{T+k}^{2}$ is given by (3). Although Baillie and Bollerslev (1992) present the expression for the conditional MSE for the $k$-steps ahead predictor of the conditional variance, the prediction error distribution for the conditional variance is not derived and, therefore, prediction intervals cannot be obtained.

It is important to notice that, as we have mentioned before, if the parameters of the GARCH model are known, the one-step ahead conditional variance is observable and, consequently, perfectly predictable. Therefore, the only uncertainty associate with the prediction of $\sigma_{T+1}^{2}$ is due to parameter uncertainty. However, if we predict the variance two or more steps ahead, then there is also uncertainty about future errors. Therefore, in this case, even if the parameters were known, the volatility cannot be perfectly predicted more than two-steps ahead. In this case, it is especially interesting to have prediction intervals for future volatilities. 


\section{Bootstrap prediction intervals}

In this section, we extend the procedure proposed in Pascual et al. (2004) for ARIMA models to obtain prediction densities of future values of returns and volatilities of series generated by GARCH processes.

Let $Y_{T}$ be a sequence of $T$ observations generated by the $\operatorname{GARCH}(1,1)$ process given by Eq. (1). The goal is to estimate directly the distribution of $y_{T+k}$ and $\sigma_{T+k}$ conditionally on the available data. Once the parameters of the model, $\theta=(\omega, \alpha, \beta)$, are estimated by $\widehat{\theta}_{T}=(\widehat{\omega}, \widehat{\alpha}, \widehat{\beta})$, the residuals are computed by $\widehat{\varepsilon}_{t}=y_{t} / \widehat{\sigma}_{t}, t=1, \ldots, T$ where $\widehat{\sigma}_{t}^{2}=\widehat{\omega}+\widehat{\alpha} y_{t-1}^{2}+$ $\widehat{\beta} \widehat{\sigma}_{t-1}^{2}, t=2, \ldots, T$, are the estimated conditional variances and $\widehat{\sigma}_{1}^{2}=\widehat{\omega} /(1-\widehat{\alpha}-\widehat{\beta})$ is the estimated marginal variance.

To implement the bootstrap technique, it is necessary to obtain bootstrap replicates $Y_{T}^{*}=$ $\left\{y_{1}^{*}, \ldots, y_{T}^{*}\right\}$ that mimic the structure of the original series. These replicates are obtained from the following recursions:

$$
\begin{aligned}
& \widehat{\sigma}_{t}^{* 2}=\widehat{\omega}+\widehat{\alpha} y_{t-1}^{* 2}+\widehat{\beta} \widehat{\sigma}_{t-1}^{* 2}, \\
& y_{t}^{*}=\varepsilon_{t}^{*} \widehat{\sigma}_{t}^{*}, \quad \text { for } t=1, \ldots, T,
\end{aligned}
$$

where $\varepsilon_{t}^{*}$ are random draws with replacement from $\widehat{F}_{T}$, the empirical distribution function of the centered residuals, and $\widehat{\sigma}_{1}^{* 2}=\widehat{\sigma}_{1}^{2}$. Once the parameters of this bootstrap series are estimated, $\widehat{\theta}_{T}^{*}=\left(\widehat{\omega}^{*}, \widehat{\alpha}^{*}, \widehat{\beta}^{*}\right)$, bootstrap forecasts of future values are obtained through the following recursions:

$$
\begin{gathered}
\widehat{\sigma}_{T+k}^{* 2}=\widehat{\omega}^{*}+\widehat{\alpha}^{*} y_{T+k-1}^{* 2}+\widehat{\beta}^{*} \widehat{\sigma}_{T+k-1}^{* 2}, \\
y_{T+k}^{*}=\varepsilon_{T+k}^{*} \widehat{\sigma}_{T+k}^{*}, \quad \text { for } k=1,2, \ldots
\end{gathered}
$$

with $\varepsilon_{T+k}^{*}$ being random draws with replacement from $\widehat{F}_{T}, y_{T}^{*}=y_{T}$ and

$$
\widehat{\sigma}_{T}^{* 2}=\frac{\widehat{\omega}^{*}}{1-\widehat{\alpha}^{*}-\widehat{\beta}^{*}}+\widehat{\alpha}^{*} \sum_{j=0}^{T-2} \widehat{\beta}^{* j}\left(y_{T-j-1}^{2}-\frac{\widehat{\omega}^{*}}{1-\widehat{\alpha}^{*}-\widehat{\beta}^{*}}\right) .
$$

Note that, in expression (7), although $\widehat{\sigma}_{T}^{* 2}$ is different for all bootstrap replicates, its value is obtained using the corresponding bootstrap parameter estimates and always the original series. Therefore, its value is small when the returns at the end of the sample period are small and large when they are large in absolute value. Consequently, $\widehat{\sigma}_{T}^{* 2}$ incorporates the variability due to parameter estimation and, at the same time, takes into account the state of the process when predictions are made.

Once we obtain a set of $B$ bootstrap replicates $\left(y_{T+k}^{*(1)}, \ldots, y_{T+k}^{*(B)}\right)$ for $y_{T+k}$, the prediction limits are defined as the quantiles of the bootstrap distribution function of $y_{T+k}^{*}$. More specifically, if $G_{y}^{*}(h)=\operatorname{Pr}\left(y_{T+k}^{*} \leqslant h\right)$ is the distribution function of $y_{T+k}^{*}$ and its Monte Carlo estimate is $G_{y, B}^{*}(h)=\#\left(y_{T+k}^{* b} \leqslant h\right) / B$, where \#(.) counts the number of cases where the condition within brackets is satisfied, then, a $100(1-\gamma) \%$ prediction interval for $y_{T+k}^{*}$ 
is given by

$$
\left[L_{y, B}^{*}(y), U_{y, B}^{*}(y)\right]=\left[Q_{y, B}^{*}\left(\frac{\gamma}{2}\right), Q_{y, B}^{*}\left(1-\frac{\gamma}{2}\right)\right],
$$

where $Q_{y, B}^{*}=G_{y, B}^{*-1}$.

We can simultaneously obtain prediction intervals for the volatility $k$ periods into the future. Given a set $\left(\widehat{\sigma}_{T+k}^{*(1)}, \ldots, \widehat{\sigma}_{T+k}^{*(B)}\right)$ of $B$ bootstrap replicates of the volatility for any horizon $k$, we proceed as before, using as prediction limits the quantiles of the bootstrap distribution function of $\widehat{\sigma}_{T+k}^{*}$. In this case, if $G_{\sigma}^{*}(h)=\operatorname{Pr}\left(\widehat{\sigma}_{T+k}^{*} \leqslant h\right)$ is the distribution function of $\widehat{\sigma}_{T+k}^{*}$ and its Monte Carlo estimate is $G_{\sigma, B}^{*}(h)=\#\left(\widehat{\sigma}_{T+k}^{* b} \leqslant h\right) / B$, a $100(1-\gamma) \%$ prediction interval for $\widehat{\sigma}_{T+k}^{*}$ is given by

$$
\left[L_{\sigma, B}^{*}(\sigma), U_{\sigma, B}^{*}(\sigma)\right]=\left[Q_{\sigma, B}^{*}\left(\frac{\gamma}{2}\right), Q_{\sigma, B}^{*}\left(1-\frac{\gamma}{2}\right)\right]
$$

where $Q_{\sigma, B}^{*}=G_{\sigma, B}^{*-1}$.

Alternatively, the bootstrap procedure just described could be also applied to construct prediction intervals conditional on the parameter estimates. This conditional bootstrap (CB) has been previously proposed by Miguel and Olave (1999) although they only focus on the construction of prediction intervals for $y_{T+k}$ and do not consider the construction of prediction intervals for future volatilities. In their proposal, the estimated parameters are kept fixed in all bootstrap forecasts of $y_{T+k}^{*}$ and $\widehat{\sigma}_{T+k}^{*}$ for $k=1,2, \ldots$. Therefore, it is not necessary to generate bootstrap replicates of the series as in (5) and the bootstrap forecasts $k$-steps ahead depend only on the resampled residuals. The recursive equations of the CB are

$$
\begin{aligned}
& \widehat{\sigma}_{T+k}^{* 2}=\widehat{\omega}+\widehat{\alpha} y_{T+k-1}^{* 2}+\widehat{\beta} \widehat{\sigma}_{T+k-1}^{* 2}, \\
& y_{T+k}^{*}=\varepsilon_{T+k}^{*} \widehat{\sigma}_{T+k}^{*}, \quad \text { for } k=1,2, \ldots,
\end{aligned}
$$

where $y_{T}^{*}=y_{T}$ and $\widehat{\sigma}_{T}^{* 2}=\widehat{\sigma}_{T}^{2}$. Since the parameter estimates are kept fixed in all bootstrap replicates of future values, the $\mathrm{CB}$ prediction intervals do not incorporate the uncertainty due to parameter estimation. Note that since in GARCH models the variance is observable one period ahead, $\widehat{\sigma}_{T+1}^{* 2}=\widehat{\omega}+\widehat{\alpha} y_{T}^{2}+\widehat{\beta} \widehat{\sigma}_{T}^{2}$ is also kept fixed in all bootstrap replicates. Consequently, $\mathrm{CB}$ does not allow to estimate the one step ahead distribution of the volatility process.

\section{Finite sample properties}

The finite sample behavior of the bootstrap procedure for prediction densities and intervals of returns and volatilities of GARCH series is now analyzed by means of Monte Carlo experiments. We generate series with the $\operatorname{GARCH}(1,1)$ model in (1) with $\omega=0.05, \alpha=0.1$ and $\beta=0.85$ and with Gaussian, Student- $t$ with 5 degrees of freedom and exponential innovations centered to have zero mean. The parameters have been chosen to resemble the parameter values often estimated when the $\operatorname{GARCH}(1,1)$ model is fitted to real series of high-frequency financial returns. With respect to the distributions, the Gaussian has been chosen because it is the most popular in empirical applications. However, it has been often 
observed in real time series that the residuals from Gaussian-GARCH $(1,1)$ models still have excess kurtosis. Consequently, it is common to assume that $\varepsilon_{t}$ has a heavy-tailed distribution like, for example, a Student- $t$ distribution. Finally, the exponential distribution is chosen to illustrate the effects on the prediction intervals of having an asymmetric conditional distribution. Results for alternative models and distributions are similar to those reported here and are available from the authors upon request. The sample sizes considered are $T=300,1000$ and 3000. The GARCH parameters are estimated by QML maximizing the Gaussian likelihood. The estimated parameters are restricted to guarantee the positivity and stationarity conditions. The corresponding intervals are constructed with nominal coverage $1-\gamma$ equal to $0.80,0.95$ and 0.99 . For each particular series generated with a particular sample size and error distribution, we generate $R=1000$ future values of $y_{T+k}$ and $\sigma_{T+k}$ and obtain $100(1-\gamma) \%$ prediction intervals for returns, denoted by $\left(L_{Y}^{*}, U_{Y}^{*}\right)$, for each of the three procedures considered. Bootstrap intervals are constructed based on $B=999$ replicates. Then, we compute the length and coverage of the interval. The coverage of each procedure is computed by $\widehat{1-\gamma_{Y}^{*}}=\#\left\{L_{y}^{*} \leqslant y_{T+k}^{r} \leqslant U_{y}^{*}\right\} / R$, where $y_{T+k}^{r}(r=1, \ldots, R)$ are future values of the variable generated previously. We also compute the coverage on the left and right tails of the distribution. Simultaneously, we compute the same quantities for future volatilities. Consequently, for each artificial series and each procedure considered, we have a measure of the length and coverage of the corresponding interval as well as of the coverage on the left and right tails of the distribution of future returns and volatilities. Then, we compute the average and standard error of the coverage and length and the average proportion of observations lying out to the left and to the right through all Monte Carlo replicates. All the results are based on 1000 replicates.

All computations have been carried out in an HP-UX C360 workstation, using Fortran 77 and the corresponding subroutines of Numerical Recipes by Press et al. (1986). In particular, Gaussian and Student- $t$ errors are generated using the subroutine "gasdev" and the corresponding transformations in each case. Exponential errors are generated using uniform random numbers generated by subroutine "rand2" and transforming them appropriately. The numerical optimization of the Gaussian log-likelihood function to obtain the QML estimates has been carried out using the subroutine "amoeba" with the maximum allowed function evaluations set equal to 5000 and the fractional convergence tolerance set equal to $10^{-6}$. This subroutine does not require analytical derivatives. Alternatively, it is possible to use computationally more efficient optimizers that use analytical derivatives of the log-likelihood. For the GARCH model, these derivatives have been calculated by Fiorentini et al. (1996). The advantage of the optimizer used in this paper is that it can be implemented even when the corresponding derivatives are not available or are difficult to program as, for example, in the asymmetric EGARCH model, in high order GARCH models or in multivariate GARCH models. In this sense, Ip et al. (2004) prove the convexity of the negative likelihood in the asymptotic sense for GARCH models. This property provides assurance for the convergence of numerical optimization algorithms for ML estimation of GARCH. Furthermore, comparing several alternative optimizers for GARCH models, they conclude that all the methods perform well for the $\operatorname{GARCH}(1,1)$ model considered in this paper.

It is important to point out that the empirical implementation of the proposed bootstrap procedure is simple and its computational costs are the usual in any resampling technique. 
Table 1

Prediction intervals for returns of $\operatorname{GARCH}(1,1)$ model with Gaussian errors

\begin{tabular}{|c|c|c|c|c|c|}
\hline $\begin{array}{l}\text { Lead } \\
\text { time }\end{array}$ & $\begin{array}{l}\text { Sample } \\
\text { size }\end{array}$ & Method & $\begin{array}{l}\text { Average } \\
\text { coverage }(\mathrm{se})^{\mathrm{a}}\end{array}$ & $\begin{array}{l}\text { Av. coverage } \\
\text { below/above }\end{array}$ & $\begin{array}{l}\text { Average } \\
\text { length }(\mathrm{se})^{\mathrm{c}}\end{array}$ \\
\hline \multirow[t]{10}{*}{1} & $T$ & Empirical & $95 \%$ & $2.5 \% / 2.5 \%$ & 3.82 \\
\hline & \multirow[t]{3}{*}{300} & STD & $94.71(0.022)$ & $2.64 / 2.65$ & $3.86(1.00)$ \\
\hline & & $\mathrm{CB}$ & $94.45(0.024)$ & $2.70 / 2.85$ & $3.85(1.03)$ \\
\hline & & PRR & $94.52(0.023)$ & $2.69 / 2.79$ & $3.84(0.945)$ \\
\hline & \multirow[t]{3}{*}{1000} & STD & $95.01(0.011)$ & $2.50 / 2.49$ & $3.84(0.846)$ \\
\hline & & $\mathrm{CB}$ & $94.86(0.014)$ & $2.51 / 2.62$ & $3.83(0.858)$ \\
\hline & & PRR & $94.85(0.014)$ & $2.53 / 2.62$ & $3.83(0.823)$ \\
\hline & \multirow[t]{3}{*}{3000} & STD & $95.01(0.009)$ & $2.50 / 2.49$ & $3.85(0.908)$ \\
\hline & & $\mathrm{CB}$ & $94.89(0.011)$ & $2.51 / 2.60$ & $3.85(0.928)$ \\
\hline & & PRR & $94.91(0.012)$ & $2.50 / 2.59$ & $3.84(0.910)$ \\
\hline \multirow[t]{10}{*}{10} & $T$ & Empirical & $95 \%$ & $2.5 \% / 2.5 \%$ & 3.90 \\
\hline & \multirow[t]{3}{*}{300} & STD & $94.44(0.025)$ & $2.78 / 2.77$ & $3.90(0.783)$ \\
\hline & & $\mathrm{CB}$ & $94.27(0.027)$ & $2.81 / 2.92$ & $3.91(0.833)$ \\
\hline & & PRR & $94.35(0.025)$ & $2.78 / 2.87$ & $3.90(0.764)$ \\
\hline & \multirow[t]{3}{*}{1000} & STD & $94.83(0.014)$ & $2.59 / 2.58$ & $3.90(0.588)$ \\
\hline & & $\mathrm{CB}$ & $94.80(0.016)$ & $2.55 / 2.65$ & $3.91(0.609)$ \\
\hline & & PRR & $94.80(0.016)$ & $2.55 / 2.65$ & $3.91(0.576)$ \\
\hline & \multirow[t]{3}{*}{3000} & STD & $94.86(0.01)$ & $2.58 / 2.56$ & $3.90(0.626)$ \\
\hline & & $\mathrm{CB}$ & $94.90(0.012)$ & $2.51 / 2.59$ & $3.92(0.646)$ \\
\hline & & PRR & $94.85(0.012)$ & $2.53 / 2.63$ & $3.92(0.638)$ \\
\hline \multirow[t]{10}{*}{20} & $T$ & Empirical & $95 \%$ & $2.5 \% / 2.5 \%$ & 3.94 \\
\hline & \multirow[t]{3}{*}{300} & STD & $94.30(0.026)$ & $2.84 / 2.86$ & $3.92(0.682)$ \\
\hline & & $\mathrm{CB}$ & $94.12(0.029)$ & $2.87 / 3.00$ & $3.93(0.762)$ \\
\hline & & PRR & $94.23(0.024)$ & $2.82 / 2.95$ & $3.93(0.713)$ \\
\hline & \multirow[t]{3}{*}{1000} & STD & $94.73(0.015)$ & $2.62 / 2.64$ & $3.92(0.447)$ \\
\hline & & $\mathrm{CB}$ & $94.71(0.017)$ & $2.59 / 2.70$ & $3.94(0.475)$ \\
\hline & & PRR & $94.77(0.016)$ & $2.55 / 2.68$ & $3.95(0.452)$ \\
\hline & \multirow[t]{3}{*}{3000} & STD & $94.77(0.010)$ & $2.60 / 2.63$ & $3.92(0.436)$ \\
\hline & & $\mathrm{CB}$ & $94.85(0.012)$ & $2.50 / 2.65$ & $3.96(0.481)$ \\
\hline & & PRR & $94.83(0.012)$ & $2.52 / 2.65$ & $3.95(0.452)$ \\
\hline
\end{tabular}

All averages have been computed through 1000 Monte Carlo replicates.

${ }^{a}$ Average coverage of prediction intervals with standard errors in parenthesis.

${ }^{b}$ Average coverage in the left and right tails of prediction densities.

${ }^{\mathrm{c}}$ Average length of predictions intervals with standard errors in parenthesis.

For example, using our own Fortran codes, the time taken by an Intel(R) Pentium computer to obtain the prediction intervals for future returns and volatilities of the IBEX-35 series analyzed in Section 5 is just 10 s.

\subsection{Prediction intervals for returns}

Table 1 reports the average coverage and the corresponding standard error (se) together with the average coverage on the left and right tails and the average length with its corresponding standard error obtained when series are generated with $\varepsilon_{t}$ Gaussian, for prediction 
Table 2

Prediction intervals for returns of $\operatorname{GARCH}(1,1)$ model with student-5 errors

\begin{tabular}{|c|c|c|c|c|c|}
\hline $\begin{array}{l}\text { Lead } \\
\text { time }\end{array}$ & $\begin{array}{l}\text { Sample } \\
\text { size }\end{array}$ & Method & $\begin{array}{l}\text { Average } \\
\text { coverage (se) }{ }^{\mathrm{a}}\end{array}$ & $\begin{array}{l}\text { Av. coverage } \\
\text { below/above }\end{array}$ & $\begin{array}{l}\text { Average } \\
\text { length }(\mathrm{se})^{\mathrm{c}}\end{array}$ \\
\hline \multirow[t]{10}{*}{1} & $T$ & Empirical & $99 \%$ & $0.5 \% / 0.5 \%$ & 5.92 \\
\hline & 300 & STD & $97.68(0.012)$ & $1.16 / 1.15$ & $4.92(1.76)$ \\
\hline & & $\mathrm{CB}$ & $98.42(0.012)$ & $0.75 / 0.81$ & $6.00(2.52)$ \\
\hline & & PRR & $98.59(0.010)$ & $0.68 / 0.73$ & $6.07(2.35)$ \\
\hline & 1000 & STD & $97.88(0.007)$ & $1.07 / 1.05$ & $4.88(1.49)$ \\
\hline & & $\mathrm{CB}$ & $98.78(0.007)$ & $0.57 / 0.66$ & $5.92(1.94)$ \\
\hline & & PRR & $98.81(0.007)$ & $0.55 / 0.64$ & $5.95(1.88)$ \\
\hline & 3000 & STD & $97.96(0.005)$ & $1.03 / 1.01$ & $4.99(1.48)$ \\
\hline & & $\mathrm{CB}$ & $98.86(0.005)$ & $0.53 / 0.61$ & $6.04(1.93)$ \\
\hline & & PRR & $98.87(0.005)$ & $0.52 / 0.61$ & $6.04(1.84)$ \\
\hline \multirow[t]{10}{*}{10} & $T$ & Empirical & $99 \%$ & $0.5 \% / 0.5 \%$ & 6.31 \\
\hline & 300 & STD & $97.56(0.012)$ & $1.22 / 1.22$ & $5.12(1.45)$ \\
\hline & & $\mathrm{CB}$ & $98.47(0.012)$ & $0.72 / 0.81$ & $0.66(2.39)$ \\
\hline & & PRR & $98.61(0.010)$ & $0.65 / 0.74$ & $6.48(2.22)$ \\
\hline & 1000 & STD & $97.73(0.008)$ & $1.14 / 1.13$ & $5.04(1.17)$ \\
\hline & & $\mathrm{CB}$ & $98.77(0.007)$ & $0.56 / 0.67$ & $6.33(1.73)$ \\
\hline & & PRR & $98.81(0.007)$ & $0.54 / 0.65$ & $6.39(1.74)$ \\
\hline & 3000 & STD & $97.82(0.006)$ & $1.10 / 1.08$ & $5.12(1.05)$ \\
\hline & & $\mathrm{CB}$ & $98.90(0.005)$ & $0.51 / 0.58$ & $6.48(1.56)$ \\
\hline & & PRR & $98.88(0.005)$ & $0.52 / 0.60$ & $6.43(1.51)$ \\
\hline \multirow[t]{10}{*}{20} & $T$ & Empirical & $99 \%$ & $0.5 \% / 0.5 \%$ & 6.51 \\
\hline & 300 & STD & $97.43(0.013)$ & $1.27 / 1.29$ & $5.19(1.36)$ \\
\hline & & $\mathrm{CB}$ & $98.41(0.013)$ & $0.76 / 0.83$ & $6.52(2.23)$ \\
\hline & & PRR & $98.50(0.011)$ & $0.71 / 0.79$ & $6.63(2.20)$ \\
\hline & 1000 & STD & $97.61(0.009)$ & $1.19 / 1.19$ & $5.13(1.02)$ \\
\hline & & $\mathrm{CB}$ & $98.71(0.008)$ & $0.58 / 0.71$ & $6.56(1.72)$ \\
\hline & & PRR & $98.75(0.007)$ & $0.57 / 0.68$ & $6.57(1.58)$ \\
\hline & 3000 & STD & $97.74(0.006)$ & $1.12 / 1.14$ & $5.18(0.784)$ \\
\hline & & $\mathrm{CB}$ & $98.85(0.005)$ & $0.51 / 0.64$ & $6.62(1.31)$ \\
\hline & & PRR & $98.86(0.005)$ & $0.52 / 0.62$ & $6.59(1.24)$ \\
\hline
\end{tabular}

All averages and standard errors have been computed through 1000 Monte Carlo replicates.

${ }^{a}$ Average coverage of prediction intervals with standard errors in parenthesis.

${ }^{b}$ Average coverage in the left and right tails of prediction densities.

${ }^{\mathrm{c}}$ Average length of predictions intervals with standard errors in parenthesis.

intervals constructed for $k=1,10$ and 20 steps ahead. Note that, for daily data, these horizons correspond approximately, to predictions made one day, two weeks and one month ahead. In Table 1 and subsequent tables, standard (STD) intervals are based on the normal approximation in (4), our proposed intervals in this paper (denoted PRR from now onwards) are bootstrap intervals that incorporate the uncertainty due to parameter estimation and, finally, $\mathrm{CB}$ intervals are bootstrap intervals conditional on parameter estimates. It is possible to observe that for Gaussian-GARCH $(1,1)$ models, all the procedures considered to construct prediction intervals for returns have similar properties for all prediction horizons and sample 
sizes considered. Furthermore, note that although the conditional distribution of $y_{T+k}$ is not normal, it seems that the normality approximation in (4) is adequate when constructing $95 \%$ prediction intervals. Also, note that the performance of the bootstrap procedures is never worse than the standard approach. Finally, when we compare PRR and CB intervals, small differences between them are observed. Therefore, introducing or not the variability due to parameter estimation does not lead to an improvement in the performance of bootstrap prediction intervals for $y_{T+k}$ in Gaussian-GARCH models.

Table 2 reports the results for the same model with $\varepsilon_{t}$ having a Student-5 distribution for $99 \%$ prediction intervals. We have chosen the $99 \%$ nominal coverage because, surprisingly, when $95 \%$ intervals are computed, all the procedures have similar properties. However, when intervals of other coverages as, for example, $80 \%$ or $99 \%$ are compared, it is possible to observe differences between the normal approximation and the bootstrap procedures. Furthermore, note that the $99 \%$ intervals could be of interest in many practical situations, for example, when computing the VaR of a given asset; see, for example, Ruiz and Pascual (2002). In Table 2, it is possible to observe that the STD average coverages and lengths are below the corresponding empirical values. The distortion does not disappear when the sample size increases. Consequently, STD intervals are clearly distorted when the error distribution is not Gaussian. As an illustration, Fig. 1 represents kernel estimates of the empirical, the PRR and the standard normal densities for a particular series of size $T=$ 1000 , for one-step ahead predictions. Note that the PRR density is remarkably close to the empirical density while the standard normal is a worse approximation, not being able to represent the higher kurtosis in the data. On the other hand, the behavior of bootstrap intervals seems appropriate with average coverages and lengths close to the empirical values. Comparing PRR and CB intervals, both have similar properties. Therefore, it seems that for symmetric distributions, introducing the uncertainty due to parameter estimation in prediction intervals is not so relevant.

All prediction intervals reported in Table 2 are based on the QML estimator obtained by maximizing the Gaussian likelihood. However, it is possible to estimate the parameters by

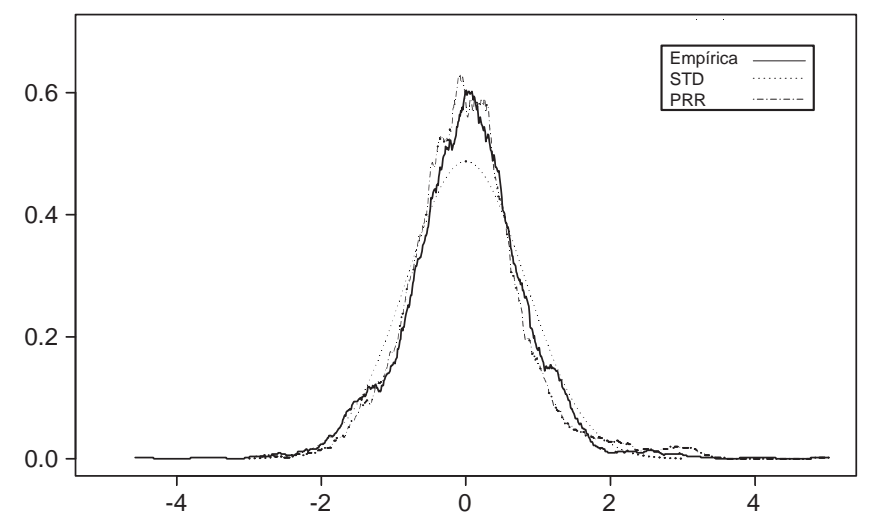

Fig. 1. Estimated kernel densities of one-step ahead predictions of returns of a particular series generated by GARCH(1,1) model with Student-5 errors and $T=1000$. 
Table 3

Prediction intervals for returns of $\operatorname{GARCH}(1,1)$ model with exponential errors

\begin{tabular}{|c|c|c|c|c|c|}
\hline $\begin{array}{l}\text { Lead } \\
\text { time }\end{array}$ & $\begin{array}{l}\text { Sample } \\
\text { size }\end{array}$ & Method & $\begin{array}{l}\text { Average } \\
\text { coverage }(\mathrm{se})^{\mathrm{a}}\end{array}$ & $\begin{array}{l}\text { Av. coverage } \\
\text { below/above }\end{array}$ & $\begin{array}{l}\text { Average } \\
\text { length }(\mathrm{se})^{\mathrm{c}}\end{array}$ \\
\hline \multirow[t]{10}{*}{1} & $T$ & Empirical & $99 \%$ & $0.5 \% / 0.5 \%$ & 4.87 \\
\hline & \multirow[t]{3}{*}{300} & STD & $96.96(0.012)$ & $0.00 / 3.04$ & $4.81(1.85)$ \\
\hline & & $\mathrm{CB}$ & $97.63(0.041)$ & $1.44 / 0.93$ & $4.88(2.15)$ \\
\hline & & PRR & $99.02(0.012)$ & $0.13 / 0.85$ & $5.04(2.04)$ \\
\hline & \multirow[t]{3}{*}{1000} & STD & $97.20(0.008)$ & $0.00 / 2.80$ & 4.88 (1.79) \\
\hline & & $\mathrm{CB}$ & $98.37(0.023)$ & $0.94 / 0.69$ & $4.93(1.97)$ \\
\hline & & PRR & 99.19 (0.009) & $0.13 / 0.67$ & 4.98 (1.90) \\
\hline & \multirow[t]{3}{*}{3000} & STD & $97.22(0.006)$ & $0.00 / 2.78$ & 4.91 (1.99) \\
\hline & & $\mathrm{CB}$ & $98.51(0.019)$ & $0.85 / 0.64$ & 4.93 (1.99) \\
\hline & & PRR & $99.20(0.008)$ & $0.15 / 0.65$ & $4.96(2.20)$ \\
\hline \multirow[t]{10}{*}{10} & $T$ & Empirical & $99 \%$ & $0.5 \% / 0.5 \%$ & 5.70 \\
\hline & \multirow[t]{3}{*}{300} & STD & $97.02(0.013)$ & $0.06 / 2.92$ & $5.00(1.60)$ \\
\hline & & $\mathrm{CB}$ & $97.76(0.029)$ & $1.32 / 0.92$ & $5.59(2.36)$ \\
\hline & & PRR & $98.25(0.017)$ & $0.88 / 0.86$ & $5.74(2.14)$ \\
\hline & \multirow[t]{3}{*}{1000} & STD & $97.31(0.010)$ & $0.04 / 2.65$ & $5.07(1.42)$ \\
\hline & & $\mathrm{CB}$ & $98.53(0.012)$ & $0.79 / 0.69$ & $5.73(1.91)$ \\
\hline & & PRR & $98.64(0.010)$ & $0.67 / 0.69$ & $5.75(1.88)$ \\
\hline & \multirow[t]{3}{*}{3000} & STD & $97.35(0.006)$ & $0.03 / 2.62$ & $5.09(1.52)$ \\
\hline & & $\mathrm{CB}$ & $98.74(0.007)$ & $0.62 / 0.64$ & $5.72(1.75)$ \\
\hline & & PRR & $98.78(0.007)$ & $0.58 / 0.63$ & $5.78(2.03)$ \\
\hline \multirow[t]{10}{*}{20} & $T$ & Empirical & $99 \%$ & $0.5 \% / 0.5 \%$ & 5.97 \\
\hline & \multirow[t]{3}{*}{300} & STD & $96.96(0.014)$ & $0.10 / 2.94$ & $5.10(1.55)$ \\
\hline & & $\mathrm{CB}$ & $97.42(0.027)$ & $1.58 / 1.00$ & $5.79(2.44)$ \\
\hline & & PRR & $98.00(0.020)$ & $1.07 / 0.93$ & $5.93(2.20)$ \\
\hline & \multirow[t]{3}{*}{1000} & STD & $97.28(0.010)$ & $0.08 / 2.64$ & $5.17(1.26)$ \\
\hline & & $\mathrm{CB}$ & $98.35(0.013)$ & $0.93 / 0.72$ & $5.97(1.83)$ \\
\hline & & PRR & $98.50(0.011)$ & $0.79 / 0.71$ & 6.03 (1.93) \\
\hline & \multirow[t]{3}{*}{3000} & STD & $97.36(0.007)$ & $0.07 / 2.57$ & $5.17(1.21)$ \\
\hline & & $\mathrm{CB}$ & $98.68(0.008)$ & $0.67 / 0.65$ & $6.01(1.73)$ \\
\hline & & PRR & $98.75(0.007)$ & $0.61 / 0.64$ & $6.03(1.80)$ \\
\hline
\end{tabular}

All averages and standard errors have been computed through 1000 Monte Carlo replicates.

${ }^{a}$ Average coverage of prediction intervals with standard errors in parenthesis.

${ }^{b}$ Average coverage in the left and right tails of prediction densities.

${ }^{\mathrm{c}}$ Average length of predictions intervals with standard errors in parenthesis.

maximizing the Student- $t$ likelihood, obtaining also an estimate of the degrees of freedom parameter. In this case, the standard prediction intervals in (4) can be constructed substituting the quantile of the standard normal distribution $z_{\gamma / 2}$ by the quantile of the corresponding Student $t$ distribution. The bootstrap intervals can also improve their performance if they are based on estimators more appropriate to the properties of the series; see Pascual et al. (2001) for an illustration of this improvement in linear ARIMA models.

Finally, Table 3 reports the results when the distribution of $\varepsilon_{t}$ is exponential. In this case, STD intervals are clearly distorted as they are not able to cope with the 
asymmetric shape of the error distribution. Comparing the resampling methods, we observe that for a sample size of 300, PRR clearly outperforms CB in the short term both in coverage and interval length, and the behavior tends to be similar as we go further into the future. As expected, the differences between PRR and CB intervals disappear with the sample size.

\subsection{Prediction intervals for volatilities}

We now analyze the performance of PRR and CB prediction intervals for future volatilities. For this purpose, we use the same Monte Carlo design used for returns. In addition, we also show the results for lead time $k=2$, since the CB technique does not provide prediction intervals for $k=1$.

Table 4 reports the results for $95 \%$ prediction intervals when series are generated with Gaussian errors. Note that, as we have mentioned before, in GARCH models, the volatility is known one-step ahead, and thus the only uncertainty associated with forecasting $\sigma_{T+1}^{2}$ is due to parameter estimation. Consequently, all the mass of the empirical distribution of $\sigma_{T+1}^{2}$ conditional on the observed series is concentrated on $\sigma_{T+1}^{2}$ and the empirical length is zero. For the same reasons, if the parameter estimates are considered as fixed, the $\mathrm{CB}$ procedure is not able to give one-step ahead prediction intervals for volatilities. Note that PRR intervals for future volatilities one-step ahead have average coverages close to the nominal values and that, as expected, their performance is better the bigger is the sample size.

When forecasting two or more steps into the future, the average coverage of CB intervals is well under the nominal value. Therefore, when the goal is to construct prediction intervals for volatilities, it is fundamental to consider the uncertainty due to parameter estimation in order to have intervals with the nominal coverage. On the other hand, the average coverage of PRR intervals is closer to the nominal for all horizons considered. Although the average length of PRR intervals is over the empirical length for sample sizes of 300 observations, it gets closer as the sample size increases. Note that the empirical distribution of future volatilities is bounded by $\omega$. Since, in practice, $\omega$ should be estimated, it is impossible to achieve exactly this bound with moderate sample sizes. In this case, the bootstrap estimate of the conditional distribution of $\sigma_{T+k}^{2}$ is smoother than the empirical distribution, and tends to have values under this bound. This leads to larger prediction intervals than the empirical ones, usually larger to the left, but with a good performance in terms of coverage. As an illustration, Fig. 2 represents the empirical, CB and PRR densities estimated for two-steps ahead predictions of volatilities generated with Gaussian errors and $T=1000$. Observe that, for moderate sample sizes $(T=1000)$, there are some distortions in the PRR bootstrap density. As we mentioned before, empirical volatilities generated by GARCH models are bounded from below while the PRR volatilities can take values below this bound. On the other hand, the CB density of future volatilities is clearly displaced to the right of the empirical density. Therefore, the PRR density provides a much better description of the distribution of future volatilities than the CB density. Finally, note that, for prediction horizons equal or greater than 2 , the shape of the volatility is asymmetric and in concordance with the shapes usually found with real data; see, for example, Andersen et al. (2001). 
Table 4

Prediction intervals for volatilities of GARCH(1,1) model with Gaussian errors

\begin{tabular}{|c|c|c|c|c|c|}
\hline $\begin{array}{l}\text { Lead } \\
\text { time }\end{array}$ & $\begin{array}{l}\text { Sample } \\
\text { size }\end{array}$ & Method & $\begin{array}{l}\text { Average } \\
\text { coverage (se) }\end{array}$ & $\begin{array}{l}\text { Av. coverage } \\
\text { below/above }\end{array}$ & $\begin{array}{l}\text { Average } \\
\text { length }(\mathrm{se})^{\mathrm{c}}\end{array}$ \\
\hline \multirow[t]{7}{*}{1} & $T$ & Empirical & $95 \%$ & $2.5 \% / 2.5 \%$ & - \\
\hline & 300 & $\mathrm{CB}$ & - & - & - \\
\hline & & PRR & $91.50(0.279)$ & $3.40 / 5.10$ & $0.65(0.667)$ \\
\hline & 1000 & $\mathrm{CB}$ & - & - & - \\
\hline & & PRR & $93.70(0.243)$ & $3.0 / 3.30$ & $0.32(0.249)$ \\
\hline & 3000 & $\mathrm{CB}$ & - & - & - \\
\hline & & PRR & $94.70(0.224)$ & $3.20 / 2.10$ & $0.18(0.174)$ \\
\hline \multirow[t]{7}{*}{2} & $T$ & Empirical & $95 \%$ & $2.5 \% / 2.5 \%$ & 0.50 \\
\hline & 300 & $\mathrm{CB}$ & $57.88(0.358)$ & $30.92 / 11.21$ & $0.56(1.01)$ \\
\hline & & PRR & $91.54(0.193)$ & $3.63 / 4.82$ & $0.96(1.25)$ \\
\hline & 1000 & $\mathrm{CB}$ & $70.52(0.274)$ & $25.69 / 3.78$ & $0.52(0.324)$ \\
\hline & & PRR & $94.19(0.122)$ & $2.91 / 2.90$ & $0.68(0.433)$ \\
\hline & 3000 & $\mathrm{CB}$ & $77.46(0.223)$ & $19.67 / 2.87$ & $0.51(0.321)$ \\
\hline & & PRR & $94.42(0.090)$ & $2.91 / 2.66$ & $0.59(0.406)$ \\
\hline \multirow[t]{7}{*}{10} & $T$ & Empirical & $95 \%$ & $2.5 \% / 2.5 \%$ & 1.33 \\
\hline & 300 & $\mathrm{CB}$ & $75.87(0.263)$ & $14.06 / 10.07$ & $1.33(2.11)$ \\
\hline & & PRR & $87.61(0.162)$ & $5.76 / 6.62$ & $1.56(2.05)$ \\
\hline & 1000 & $\mathrm{CB}$ & $89.52(0.099)$ & $6.56 / 3.92$ & $1.34(0.733)$ \\
\hline & & PRR & $92.57(0.074)$ & $3.91 / 3.52$ & $1.41(0.756)$ \\
\hline & 3000 & $\mathrm{CB}$ & $93.26(0.04)$ & $3.80 / 2.94$ & $1.37(0.715)$ \\
\hline & & PRR & $94.17(0.03)$ & $2.96 / 2.87$ & $1.39(0.750)$ \\
\hline \multirow[t]{7}{*}{20} & $T$ & Empirical & $95 \%$ & $2.5 \% / 2.5 \%$ & 1.62 \\
\hline & 300 & $\mathrm{CB}$ & $75.85(0.254)$ & $13.68 / 10.46$ & $1.58(2.23)$ \\
\hline & & PRR & $85.73(0.167)$ & $6.80 / 7.47$ & $1.79(2.15)$ \\
\hline & 1000 & $\mathrm{CB}$ & $89.64(0.091)$ & $6.18 / 4.17$ & $1.62(0.805)$ \\
\hline & & PRR & $91.83(0.074)$ & $4.35 / 3.81$ & $1.68(0.807)$ \\
\hline & 3000 & $\mathrm{CB}$ & $93.35(0.040)$ & $3.27 / 1.79$ & $1.65(0.728)$ \\
\hline & & PRR & $93.90(0.035)$ & $3.11 / 2.99$ & $1.66(0.737)$ \\
\hline
\end{tabular}

All averages and standard errors have been computed through 1000 Monte Carlo replicates.

${ }^{a}$ Average coverage of prediction intervals with standard errors in parenthesis.

${ }^{b}$ Average coverage in the left and right tails of prediction densities.

${ }^{\mathrm{c}}$ Average length of predictions intervals with standard errors in parenthesis.

The results in Table 4 show that in finite samples even if they are large, it is necessary to introduce the variability of the parameter estimates in order to obtain prediction intervals for the volatility with coverages close to the nominal values. We have also compared the properties of prediction intervals for volatilities when the series are generated by GARCH models with Student-5 and exponential errors with results similar to the ones reported in Table 4. Consequently, we do not report these results to save space although they are available upon request. 


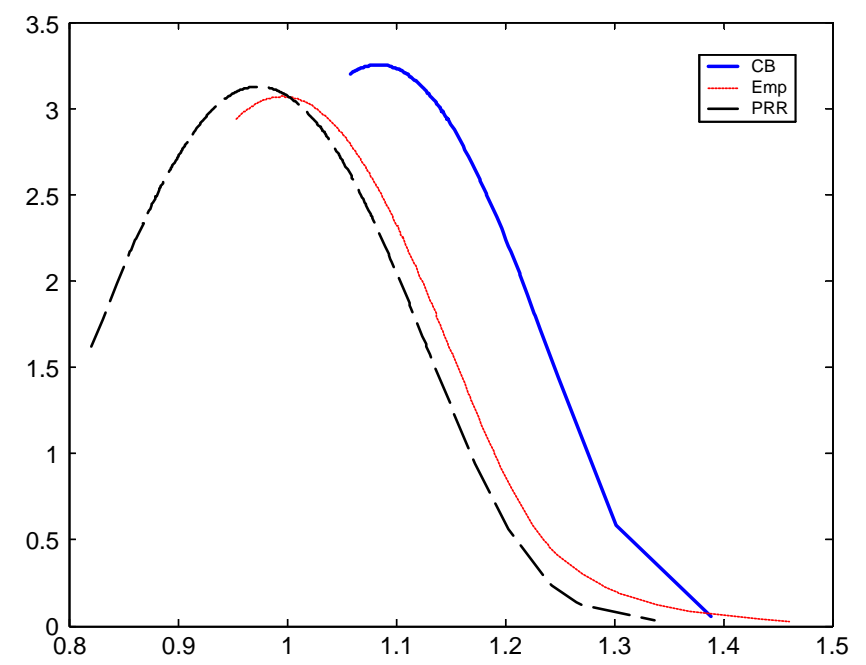

Fig. 2. Estimated kernel densities of two-steps ahead predictions of volatilities of a particular series generated by GARCH(1,1) models with Gaussian errors and $T=1000$.

\section{An application with real data}

In this section, we apply the PRR procedure to construct prediction intervals for returns and volatilities of the Madrid Stock Market index IBEX-35. The estimation of the GARCH model used for the prediction of future returns and volatilities is based on daily closing prices of the IBEX-35 observed from 2 January 1996 to 3 March 2000, with a total of 1045 observations. As usual, daily percentage returns are obtained as first differences of logarithms scaled by multiplying by 100 , i.e., $R_{t}=100 \log \left(P_{t} / P_{t-1}\right)$, where $P_{t}$ denotes the closing price at day $t$. The original series of returns has been filtered previous to its analysis to clean a small although significant autocorrelation of order one and the effect of two extraordinary events that occur in Spain during the period of time considered. In particular, the stock market had a sharp decline on the 4th of March of 1996 when the Partido Popular (PP) won the elections in Spain by a narrow margin and on the 4th of January 1999 when the Euro was introduced; see Carnero et al. (2004) for the effects of outliers on the estimation of the conditional heteroscedasticity. The estimated MA(1) model with interventions is given by

$$
\begin{aligned}
R_{t}= & \underset{(0.046)}{0.1188}+\hat{\mathrm{a}}_{t}+\underset{(0.047)}{0.1037} \hat{\mathrm{a}}_{t-1}-\underset{(0.273)}{5.2694} D_{1 t}+\underset{(0.287)}{5.8184} D_{2 t} \\
& -\underset{(0.134)}{6.9508} D_{3 t}-\underset{(0.487)}{3.2550} D_{4 t}
\end{aligned}
$$

where the standard errors appear in parenthesis. The variables $D_{1 t}$ and $D_{2 t}$ are pulse dummy variables that take value 1 on the 4th of March of 1996 and on the 4th of January 1999, respectively. The last two dummy variables are due to extreme market reactions to external effects. Observe that the estimated MA parameter is rather small, 0.1037 , and, consequently, apart from the outliers, the original and filtered series of returns are very similar. There- 
Table 5

Sample moments of residuals from MA(1) model with interventions

\begin{tabular}{|c|c|c|c|c|c|c|c|}
\hline Sample size & Mean & Median & S.D. & Skewness & Kurtosis & Max. & Min. \\
\hline 1045 & -0.0001 & -0.0019 & 1.3697 & $-0.3098^{*}$ & $5.9578^{*}$ & 5.7889 & -7.1571 \\
\hline Autocorrelations & $r(1)$ & $r(2)$ & $r(3)$ & $r(4)$ & $r(5)$ & $r(10)$ & $r(20)$ \\
\hline$y_{t}$ & -0.007 & -0.066 & -0.020 & -0.005 & 0.041 & 0.066 & -0.058 \\
\hline [s.e.] & [0.047] & [0.044] & {$[0.044]$} & [0.047] & [0.045] & [0.048] & [0.042] \\
\hline$y_{t}^{2}$ & $0.273^{*}$ & $0.225^{*}$ & $0.224^{*}$ & $0.274^{*}$ & $0.228^{*}$ & $0.304^{*}$ & $0.179^{*}$ \\
\hline [s.e.] & {$[0.031]$} & {$[0.031]$} & {$[0.031]$} & [0.031] & [0.031] & [0.031] & [0.031] \\
\hline
\end{tabular}

${ }^{*}$ Significant values at $5 \%$ level.

Table 6

Sample moments of standardized residuals

\begin{tabular}{|c|c|c|c|c|c|c|c|}
\hline Sample size & Mean & Median & S.D. & Skewness & Kurtosis & Max. & Min. \\
\hline $\begin{array}{l}1045 \\
\text { Series/Lag }\end{array}$ & $\begin{array}{l}0.0067 \\
r(1)\end{array}$ & $\begin{array}{l}0.0026 \\
r(2)\end{array}$ & $\begin{array}{l}0.9932 \\
r(3)\end{array}$ & $\begin{array}{l}-0.2257^{*} \\
r(4)\end{array}$ & $\begin{array}{l}3.1470 \\
r(5)\end{array}$ & $\begin{array}{l}2.6983 \\
r(10)\end{array}$ & $\begin{array}{l}-3.8791 \\
r(20)\end{array}$ \\
\hline $\begin{array}{l}\widehat{\varepsilon}_{t} \\
{[s . e .]} \\
\widehat{\varepsilon}_{t}^{2} \\
{[\text { s.e. }]}\end{array}$ & $\begin{array}{c}-0.007 \\
{[0.030]} \\
-0.007 \\
{[0.031]}\end{array}$ & $\begin{array}{c}-0.029 \\
{[0.031]} \\
0.026 \\
{[0.031]}\end{array}$ & $\begin{array}{c}-0.002 \\
{[0.032]} \\
0.039 \\
{[0.031]}\end{array}$ & $\begin{array}{c}0.026 \\
{[0.031]} \\
0.026 \\
{[0.031]}\end{array}$ & $\begin{array}{c}0.013 \\
{[0.030]} \\
-0.003 \\
{[0.031]}\end{array}$ & $\begin{array}{c}0.028 \\
{[0.030]} \\
-0.014 \\
{[0.031]}\end{array}$ & $\begin{array}{c}-0.032 \\
{[0.031]} \\
0.010 \\
{[0.031]}\end{array}$ \\
\hline
\end{tabular}

* Significant values at $5 \%$ level.

fore, we expect that the results of the Monte Carlo experiments reported in the previous section, can be applied to the series of the IBEX-35 residuals, which from now on, will be denoted by $y_{t}$. Table 5 reports the sample moments of $y_{t}$. The estimated kurtosis coefficient is significantly larger than 3 showing that the return distribution is leptokurtotic. Table 5 also contains the sample autocorrelations of returns and their squares. The standard errors of sample autocorrelations of returns are corrected by ARCH effects as suggested by Diebold (1986). The autocorrelation of returns are not significant although their squares are significantly autocorrelated.

The results reported in Table 5 suggest that the filtered IBEX-35 returns can be conditionally heteroscedastic. Consequently, we fit a $\operatorname{GARCH}(1,1)$ model to them. Doornik and Ooms (2003) point out that when, as in our case, dummy variables are added to the mean of a GARCH model, the likelihood can be bimodal. To check that we are not facing this problem in our data, we have used a battery of starting values for the optimizer of the likelihood. The estimated model is

$$
\widehat{\sigma}_{t}^{2}=\underset{(0.011)}{0.0209}+\underset{(0.018)}{0.1060} y_{t-1}^{2}+\underset{(0.020)}{0.8866} \widehat{\sigma}_{t-1}^{2} .
$$

The sample moments of the standardized residuals, $\widehat{\varepsilon}_{t}$, appear in Table 6 , where it can be observed that the estimated GARCH model is able to represent adequately the dynamic structure of the volatility process. Although the excess kurtosis parameter is not significantly 

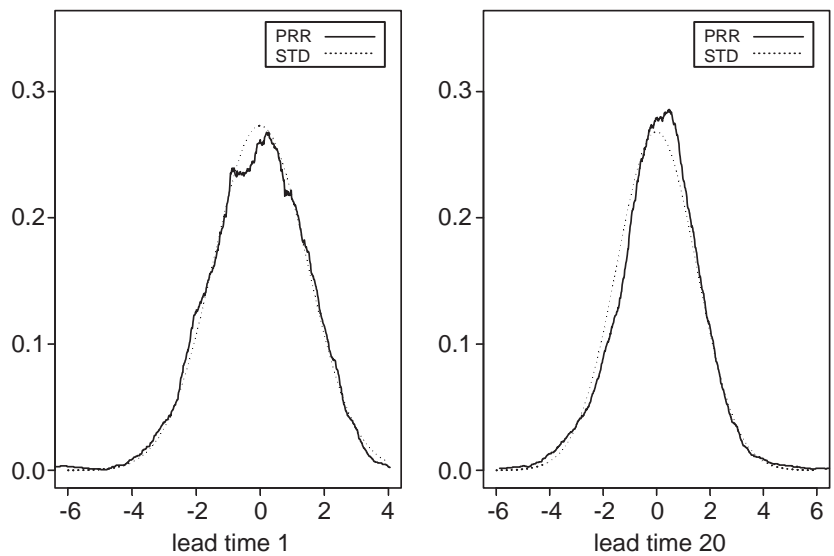

Fig. 3. Estimated kernel densities of one- and 20-step ahead predictions of returns.
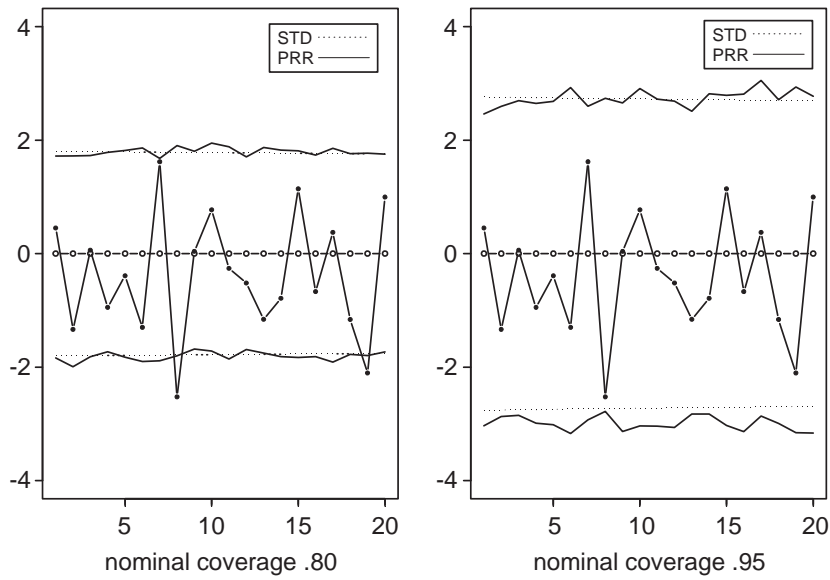

Fig. 4. Prediction intervals of future returns together with real observations $(\bullet)$ and point linear predictions (o).

different from 3, the skewness coefficient is significantly different from zero ( $p$-value is 0.04). The Jarque-Bera statistic for normality equals 9.2996 ( $p$-value is 0.0095 ). Therefore, the standardized residuals are not Gaussian.

Next, we apply the PRR procedure to obtain out-of-sample prediction intervals of future returns $y_{T+k}$ from 4 March 2000 until 31 March 2000. The estimated bootstrap densities for $k=1$ and 20 steps ahead, that correspond to predictions made one-day and one-month ahead, appear in Fig. 3 where it is possible to observe the asymmetric shape previously observed in the standardized returns. Using the bootstrap densities, we construct $80 \%$ and 95\% PRR prediction intervals for $y_{T+k}$ that have been plotted in Fig. 4 together with the point linear prediction of $y_{T+k}$ (zero for all horizons), the observed returns and the corresponding prediction intervals constructed using the normal approximation. Once more, we can see the asymmetry of the standardized returns, providing prediction intervals slightly larger to 

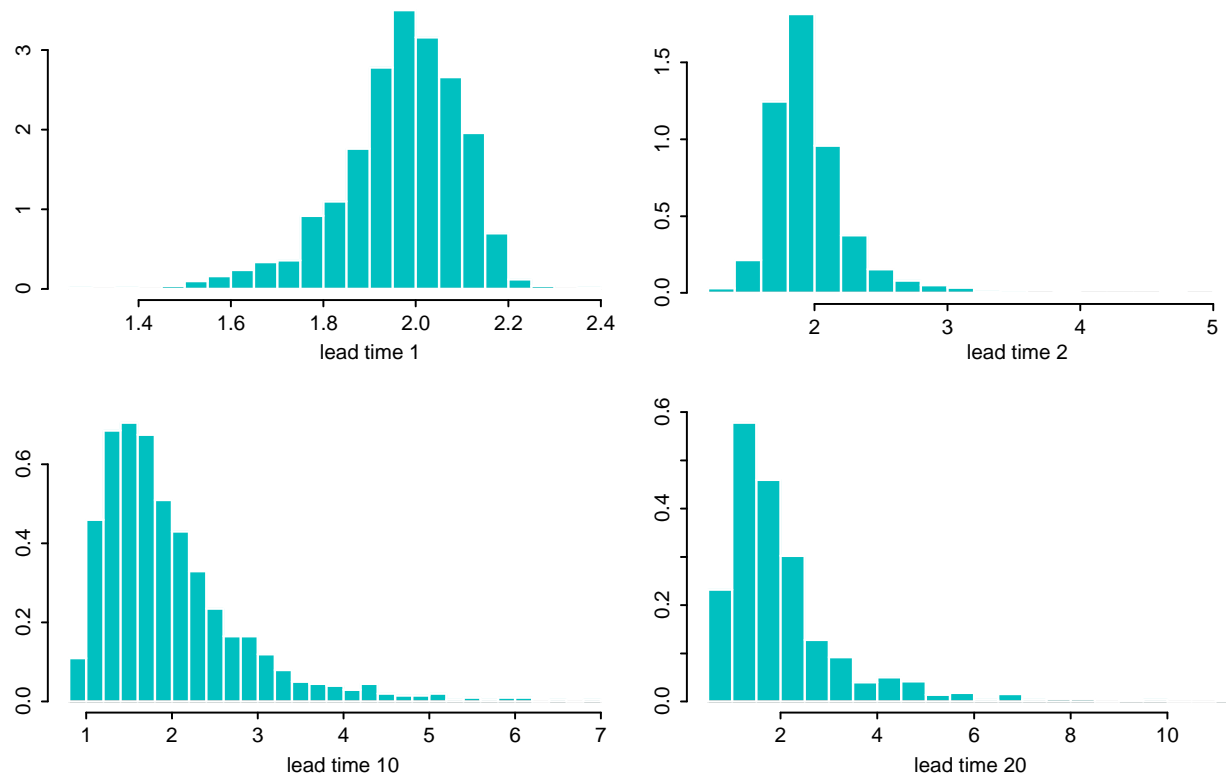

Fig. 5. Histograms of bootstrap predictions of future volatilities of returns.

the left than those obtained by standard methods.

Finally, we construct bootstrap prediction intervals for future volatilities. In Fig. 5, we plot the histograms for the bootstrap predictions of volatilities 1,2, 10 and 20 steps ahead into the future. Note that the shape of volatility predictions is asymmetric and similar to the bootstrap densities obtained in the Monte Carlo experiments and represented in Fig. 2. A common approach for judging prediction intervals is to check whether they contain the subsequent realizations of volatility with the desired coverage. However, as volatility is not directly observed, this approach is not immediately applicable for prediction interval evaluation. Different measures of volatility have been used in the literature to check whether GARCH models provide good forecasts of volatility. Andersen and Bollerslev (1998) propose to use the realized volatility as a measure of volatility. They show that, if logarithmic prices evolve as a diffusion and if returns are sampled sufficiently frequently, then the realized volatility is an efficient proxy to volatility and becomes arbitrarily close to the true volatility as the sampling frequency increases. However, on the other hand, market microstructure can have a large impact on ultra-high frequency returns; see, for example, Andersen et al. (2003). In this paper, we compute realized volatilities using one-minute returns based on tick by tick prices observed from 4 March 2000 to 31 March 2000. The volatility at day $t$ is estimated by the sum of the corresponding squared returns during day $t$, i.e. $\sigma_{t}^{2}=R_{t, 1}^{2}+\cdots+R_{t, n}^{2}$, where $n$ is the number of observations available at day $t$ which is approximately 500 .

Fig. 6 shows point linear predictions of volatility together with the corresponding realized volatilities and $80 \%$ and $95 \%$ PRR prediction intervals. These intervals have been obtained reestimating the parameters each time a new observation is available and constructing the 

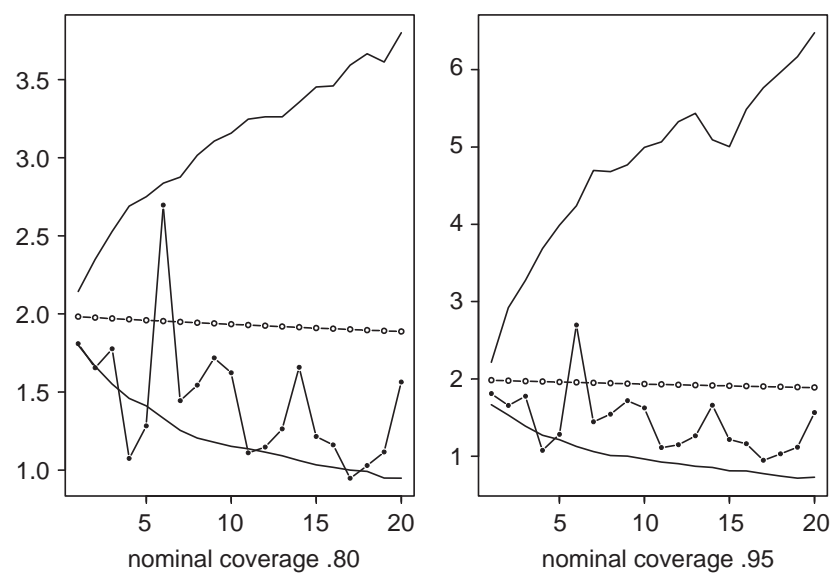

Fig. 6. Bootstrap prediction intervals of future volatilities together with realized volatilities computed using one-minute returns $(\bullet)$ and point predictions $(\circ)$.
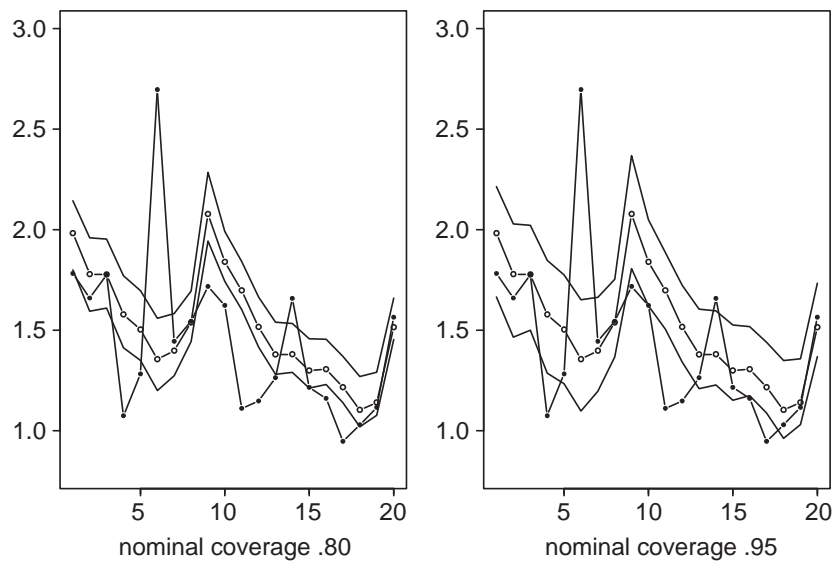

Fig. 7. One-step ahead bootstrap prediction intervals for future volatilities together with realized volatilities computed using one-minute returns $(\bullet)$ and point predictions $(\circ)$.

corresponding one-step ahead bootstrap interval. In this figure, we can see how the proposed resampling scheme gives good prediction intervals for both $80 \%$ and $95 \%$ in the sense that the $80 \%$ intervals leave 5 future values out when it is supposed to leave 4 and the $95 \%$ intervals leave 1 out that corresponds exactly with the nominal coverage. The empirical results are in concordance with the simulation results reported in Table 4 that shows that the empirical coverages of the intervals for future volatilities could be under the corresponding nominal coverages.

Finally, Fig. 7 plots the realized volatility estimated using one-minute returns together with the one-step ahead bootstrap prediction intervals. These intervals have been obtained re-estimating the parameters each time a new observation is available and constructing the 
corresponding one-step ahead bootstrap interval. In this case, the coverage is well under the nominal. However, this result is in concordance with our previous findings. Remember that, in the simulation experiments, we have seen that the bootstrap and empirical densities are closer when the prediction horizon increases. As we have mentioned before, this lack of adequacy for short horizons could be related more with the way GARCH models represent the volatility than with the bootstrap procedure. When the volatility is predicted one-step ahead, the only uncertainty associated with this prediction is introduced through the parameter uncertainty and, looking at Fig. 7, it seems rather clear that the uncertainty about future volatilities should be bigger.

\section{Conclusions}

In this paper, we have extended to GARCH processes the bootstrap procedure originally introduced by Pascual et al. (2004) to construct prediction intervals for ARIMA models. The bootstrap prediction intervals proposed incorporate the uncertainty due to parameter estimation and do not rely on any assumption on the error distribution. Furthermore, incorporating the variability of the estimators, we can construct prediction intervals not only for future returns but also for volatilities.

We analyze the finite sample behavior of the proposed bootstrap procedure by means of extensive Monte Carlo experiments. The results of these experiments show that the standard prediction intervals for returns built treating the error distribution as if they were normal for any prediction horizon are adequate as far as the model is conditionally normal. However, it has often been observed in empirical applications that the conditional distribution of the errors of GARCH models is leptokurtic; see, for example, Bollerslev et al. (1994) and the references therein. Standard prediction intervals for returns are not able to deal with non-Gaussian errors while bootstrap intervals do. The results of the Monte Carlo experiments also show that incorporating or not the variability due to parameter estimation makes no difference when building prediction intervals for returns as far as the conditional distribution is symmetric. However, to construct prediction intervals for future volatilities, it is necessary to introduce the uncertainty due to parameter estimation in order to have intervals with coverages close to the nominal values. Although all the results presented in this paper refer to the $\operatorname{GARCH}(1,1)$ model, the extension to $\operatorname{GARCH}(\mathrm{p}, \mathrm{q})$ models is straightforward.

Finally, it is important to mention that the proposed bootstrap prediction intervals for future volatilities are too wide when compared with empirical intervals. As we have noted before, this effect could be due to the way GARCH models specify the volatility that is observable one-step ahead. In this sense, Andersen (2000) shows that volatility diffusion models often used in finance render discrete-time strong-form ARCH based models invalid because it is impossible for a discrete return to serve as a sufficient statistic for the innovation to the volatility process. Alternatively, volatility can be modelled by stochastic volatility (SV) models, as proposed, for example, by Harvey et al. (1994), which represent the volatility as an unobservable latent process. It could be worth to investigate the performance of the PRR procedure in the context of SV models. 


\section{Acknowledgements}

We are very grateful for their helpful comments by three anonymous referees, the editor Stephen Pollock and seminar participants at the Universities of Valladolid, New South Wales and Canterbury and the June 2001 Time Series Workshop of Arrabida, the September 2001 International Conference on Modelling Volatility (Perth) and the June 2002 International Symposium on Forecasting (Dublin). We are also grateful to Gregorio Serna for providing the data set analyzed in this paper and to Dolores Redondas for helping us with the figures. Financial support was provided by projects DGES PB96-0111 and BEC2002-03720 from the Spanish Government and Cátedra de Calidad from BBVA.

\section{References}

Andersen, T.G., 2000. Some reflections on analysis of high-frequency data. J. Business Econom. Statist. 18, $146-153$.

Andersen, T.G., Bollerslev, T., 1998. Answering the skeptics: yes, standard volatility models do provide accurate forecasts. Internat. Econom. Rev. 39, 885-905.

Andersen, T.G., Bollerslev, T., Diebold, F.X., Labys, P., 2001. The distribution of realized exchange rate volatility. J. Amer. Statist. Assoc. 96, 42-55.

Andersen, T.G., Bollerslev, T., Diebold, F.X., Labys, P., 2003. Modelling and forecasting volatility. Econometrica 71, 579-625.

Baillie, R.T., Bollerslev, T., 1992. Prediction in dynamic models with time dependent conditional variances. J. Econometrics 52, 91-113.

Bollerslev, T., 1986. Generalized autoregressive conditional hteroskedasticity. J. Econometrics 31, 307-327.

Bollerslev, T., 2001. Financial econometrics: past developments and future challenges. J. Econometrics 100, 41-51.

Bollerslev, T., Engle, R.F., Nelson, D.B., 1994. ARCH. In: Handbook of Econometrics, vol. 4. pp. 2959-3038.

Carnero, M.A., Peña, D., Ruiz, E., 2004. Spurious and hidden volatility. WP, Universidad Carlos III de Madrid.

Diebold, F.X., 1986. Empirical modeling of exchange rate dynamics. Lectures Notes in Economics and Mathematical Systems. Springer, Berlin.

Doornik, J.A., Ooms, M., Multimodality in the GARCH regression model. Mimeo, Nuffield College, University of Oxford, 2003.

Engle, R.F., 2001. Financial econometrics: a new discipline with new methods. J. Econometrics 100, 53-56.

Engle, R.F., Patton, A., 2001. What good is a volatility model. Quantitative Finance 1, 237-245.

Fiorentini, G., Calzolari, G., Panattoni, L., 1996. Analytic derivatives and the computation of GARCH estimates. J. Appl. Econometrics 11, 399-417.

Harvey, A.C., Ruiz, E., Shephard, N., 1994. Multivariate stochastic variance models. Rev. Econom. Stud. 61, 247-264.

Ip, W.C., Wong, H., Pan, J.Z., Li, D.F., 2004. The asymptotic convexity of the negative likelihood function of GARCH models. Comput. Statist. Data Anal., in press.

Jacquier, E., Polson, N.G., Rossi, P.E., 1994. Bayesian analysis of stochastic volatility models (with discussion). J. Business Econom. Statist. 12, 371-417.

Miguel, J.A., Olave, P., 1999. Bootstrapping forecast intervals in ARCH models. Test 8, 345-364.

Nelson, D.B., 1990. Stationary and persistence in the GARCH(1,1) model. Econom. Theory 6, 318-344.

Pascual, L., Romo, J., Ruiz, E., 2001. Effects of parameter estimation on prediction densities: a bootstrap approach. Internat. J. Forecasting 17, 83-103.

Pascual, L., Romo, J., Ruiz, E., 2004. Bootstrap predictive inference for ARIMA processes. J. Time Ser. Anal. 25, 449-465.

Press, W.H., Flannery, B.P., Teukolsky, S.A., Vetterling, W.T., 1986. Numerical Recipes. Cambridge University Press, Cambridge.

Ruiz, E., Pascual, L., 2002. Bootstrapping financial time series. J. Econom. Surv. 16, 271-300. 
Shephard, N.G., 1996. Statistical aspects of ARCH and stochastic volatility. In: Cox, D.R., Hinkley, D.V., BarndorffNielsen, O.E. (Eds.), Time Series Models in Econometrics, Finance and Other Fields. Chapman \& Hall, London. Tsay, R.S., 2002. Analysis of Financial Time Series. Wiley, New York. 